\title{
Impact-parameter-dependent multiple ionisation probabilities for fast proton-neon collisions*
}

\author{
H Sharabati†, K Bethge $\ddagger, J$ Ullrich $\ddagger, R$ Dörner $\ddagger, \mathrm{R}$ E Olson $§$, \\ $\mathrm{V}$ Dangendorf $\ddagger, \mathrm{R}$ Koch $\ddagger$ and $\mathrm{H}$ Schmidt-Böcking $\ddagger$ \\ $\uparrow$ University of Jordan, Amman, Jordan \\ ¥Institut für Kernphysik, Universität Frankfurt, D-6000 Frankfurt, Federal Republic of \\ Germany \\ §esellschaft für Schwerionenforschung, D-6100 Darmstadt, Federal Republic of Germany
}

Received 15 February 1990

\begin{abstract}
Absolute multiple ionisation probabilities in dependence of the impact parameter $b$ have been measured for 0.5 and $1.0 \mathrm{MeV}$ proton on neon collisions in an impact parameter regime of $b \leqslant r_{\mathrm{K}}$, where $r_{\mathrm{K}}$ is the expectation value for the $\mathrm{Ne} \mathrm{K}$-shell radius.

The analysis of the experimental data within the independent-particle model provided $b$-dependent absolute $\mathrm{K}$ and L-shell ionisation probabilities $P_{\mathrm{K}}(b)$ and $P_{\mathrm{L}}(b)$. The $P_{\mathrm{L}}(b)$ are in fair agreement with results of classical many-body calculations (CTMC), the $P_{K}(b)$ are well described in the semiclassical approximation (SCA).
\end{abstract}

\section{Introduction}

Multiple ionisation (MI) of rare-gas atoms in collisions with fast charged projectiles is a complicated mechanism due to the many-body nature of the interaction. For target atoms with more than two electrons present, a complete quantum mechanical as well as classical calculation of total MI cross sections, including the electron-electron interaction, is a formidable task and beyond the capability of present supercomputers. It has been shown however, theoretically (Olson 1979, 1988, Becker et al 1984, Åberg et al 1982, Horbatsch and Dreizler 1985, Horbatsch 1986) as well as experimentally (Cocke 1979, Müller et al 1986, Kelbch et al 1984, Ullrich et al 1986) for a large collection of different collision systems at moderately high projectile energies $E_{\mathrm{P}}$, that total MI cross sections can be reasonably well described within the independent-particle approximation (IPA, McGuire and Weaver 1977) which reduces the description of the many-body ionisation process to a single particle problem and classical statistics is applied to obtain MI cross sections.

Up to now, experimental studies concentrated on total MI cross section measurements, with few experimental data on projectile angular differential (Schuch et al 1988, Kelbch et al 1988, Kamber et al 1988, Giese and Horsdal 1988) or recoil-ion energy differential MI cross sections (Levin et al 1987, Grandrin et al 1988, Olson et al 1987, Ullrich et al 1988, Dörner et al 1989) having been reported. In these differential measurements large impact parameter collisions with small transverse momentum

* Supported by BMFT.

|| Humboldt-Award Fellow. Permanent address: University of Missouri-Rolla, Rolla, MO 65401, USA. 
transfers to the recoil-ion or to the projectile of $p_{\perp} / p_{0} \leqslant 10^{-3}$ were investigated $\left(p_{\perp}\right.$ is the final projectile or recoil-ion transverse momentum, $p_{0}$ is the initial projectile longitudinal momentum). In addition, in many cases the incoming ions were highly charged. Due to polarisation of the target electron cloud by the Coulomb force of a highly charged projectile and to direct interaction of the projectile with the ionised electrons at very large $b$ it was found to be essential to include the electron-nuclei interactions into theory in order to understand the collision dynamics (Ullrich et al 1989, Dörner et al 1989, Olson et al 1989). According to classical calculations (Olson 1988, Horbatsch 1989), which are in general agreement with experimental results, this interaction disturbs strongly the deflection function $b(\vartheta)(\vartheta$ is the projectile laboratory scattering angle).

Thus we have concentrated on small impact parameter collisions in order to report the first experimental $b$-dependent $\mathrm{MI}$ probabilities at small impact parameters of $0.01 a_{0} \leqslant b \leqslant 0.2 a_{0}$ for a relatively small perturbation by the singly charged projectile. In contrast to the previous measurements, the projectile scattering angle regime investigated is beyond $1 \mathrm{mrad}$ where the deflection of the projectile $\vartheta$ is dominated by the interaction with the target nucleus. Therefore, the scattering can be described by a well defined deflection function $b(\vartheta)$ in a screened Coulomb potential. The perturbation by the singly charged projectile is so small that polarisation of the target electron cloud should be of minor importance. Therefore, impact-parameter-dependent MI probabilities could be analysed in the framework of the IPM and compared with theoretical results.

\section{Experiment}

The energy-analysed proton beam from the $2.5 \mathrm{MV}$ van de Graaff accelerator of the Institut für Kernphysik (University of Frankfurt) was well defined by two collimators over a length of $3.5 \mathrm{~m}$. A third one was placed directly in front of the interaction region in order to reduce slit scattering by the beam defining collimators. After passing the gas target cell, which was differentially pumped in three stages, the projectile deflection in a plane perpendicular to the beam axis has been measured by a two-dimensional position-sensitive parallel plate avalanche detector (PPAD, Gaukler et al 1977). The undeflected particles were dumped on a mask in front of the detector. The target pressure in the innermost gas cell of about $3 \times 10^{-1} \mathrm{~Pa}$ was measured by a Baratron manometer and controlled by a servo leak valve. Pressures in the second stage of about $8 \times 10^{-3} \mathrm{~Pa}$ and of $1 \times 10^{-4} \mathrm{~Pa}$ in the third guaranteed a well localised gas target. Slit scattering contributions and scattering from the residual gas could be minimised to a negligibly small amount. The recoil ions, produced in the interaction region, were accelerated in an electric field of about $300 \mathrm{~V} \mathrm{~cm}^{-1}$, drifted in a field free tube with a length about twice the extraction length to optimise the time resolution and finally were detected by a channeltron detector. A coincidence between the signals of the scattered projectiles and the recoil ions was used to determine the recoil-ion charge state since their time-of-flight (TOF) in the accelerating field and in the drift tube depends on their charge state. The details of this spectrometer has been described elsewhere (Kelbch et al 1984).

In contrast to previous measurements, where the projectile scattering angle $\vartheta$ was either not defined or very small, the recoil-ion energies corresponding to the large $\vartheta$ investigated in this experiment are considerably higher, i.e. larger than $20 \mathrm{eV}$. Therefore, 
and due to the limited accelerating voltage $V_{\mathrm{a}}$ (figure 1) which can be applied in the recoil-ion spectrometer, the detection solid angle is a function of the recoil-ion azimuthal scattering angle $\varphi_{\mathrm{R}}$, its energy $E_{\mathrm{R}}$ and its charge state $q$, illustrated in figure 1 for isotropic scattering and two different recoil energies: only recoil ions with a sufficiently small momentum transverse $\left(p_{y}\right)$ compared to that parallel $\left(p_{x}\right)$ to the electric field can pass the defining aperture. Furthermore, ions being scattered parallel or antiparallel to the accelerating electric field have different times of flight (TOF) which gives rise to a double peak structure of the time peaks for each charge state, shown in figure 2 for $0.5 \mathrm{MeV} \mathrm{He}^{+}$impact on $\mathrm{Ne}$. The difference in the ToF between the double peaks $(\Delta t)$ directly reflects the recoil-ion energy and therefore depends on the projectile scattering angle $\vartheta$, as visualised in figure 3 as logarithmic contour plots in the two-dimensional TOF versus $\vartheta$ spectrum. The time difference between target ions being scattered along (line b) or against (line a) the electric field increases with increasing projectile scattering angle $\vartheta$ and decreases with increasing recoil-ion charge state $q$ since ions with a higher $q$ are accelerated more strongly.

To control the recoil-ion detection solid angle as a function of the recoil-ion charge state and energy (projectile scattering angle $\vartheta$ ), the scattering plane, namely the

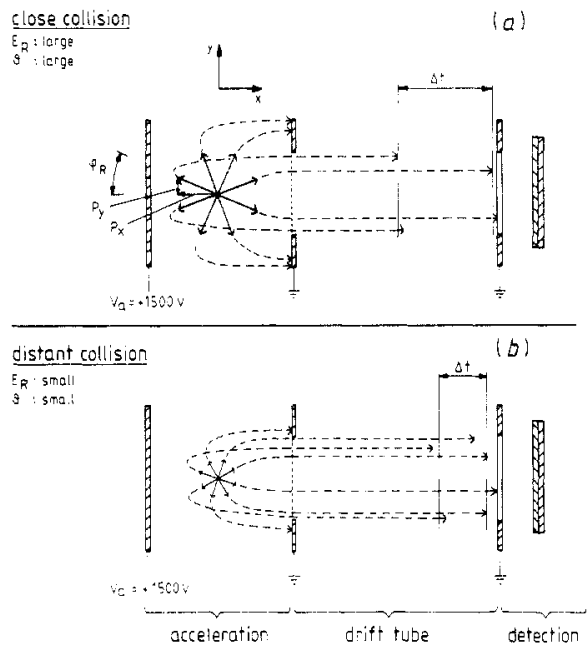

Figure 1. Illustration of the influence of the scattering kinematics on the solid angle of the recoil-ion detection system for $(a)$ a large impact parameter collision and $(b)$ a close encounter.

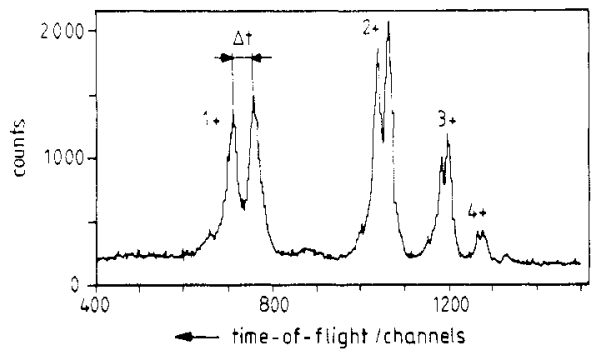

Figure 2. Time-of-flight (TOF) spectrum for $0.5 \mathrm{MeV} \mathrm{He}^{+}$on $\mathrm{Ne}$ collisions: two time peaks for each charge state are observed due to kinematical effects. 


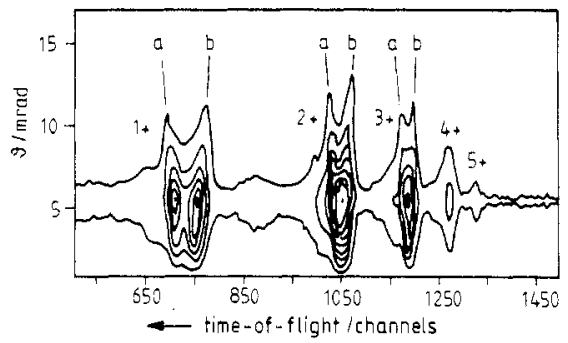

Figure 3. TOF plotted against the projectile scattering angle $\vartheta$ : the time difference $\Delta t$ between recoil ions scattered parallel or antiparallel to the electric extraction field in the spectrometer increases with the projectile scattering angle (momentum transfer to the recoil ion) and decreases for the higher charge states. Lines $a$ and $b$ indicate the expected ToF position for parallel and antiparallel scattering.

recoil-ion azimuthal emission angle $\varphi_{R}$, has to be determined. This was obtained by a $\varphi_{\mathrm{P}}$ angle measurement of the projectile using the two-dimensional position sensitive PPAD according to the identity $\varphi_{R}=180^{\circ}-\varphi_{P}$, which is valid for a two-body collision and should be a good approximation for the close encounters investigated. By setting different software windows on $\varphi_{\mathrm{P}}$ for each scattering angle $\vartheta$ (recoil energy) the recoil-ion detection efficiency could be controlled exactly. This is shown in figure 4 , where two TOF versus $\vartheta$ spectra are displayed measured in coincidence either to $\varphi_{R}$ parallel $\left(0^{\circ} \pm 10^{\circ}\right.$ ) (figure $4(a)$ ) or antiparallel to the accelerating field $\left(180^{\circ} \pm 10^{\circ}\right)$ (figure $4(b)$ ). Now, only one time peak for each charge state is visible, the small structure left of each charge state is due to ionisation of ${ }^{22} \mathrm{Ne}$ isotopes present in the $\mathrm{Ne}$ gas target. Note: the detection efficiencies 'along' and 'against' the electric field are quite different as is obvious from figures 2 and 3.

As mentioned above, the scattered projectiles were detected in a PPAD using a 'wedge-and-strip' geometry of the anode to obtain the two-dimensional position resolution. The gas counter had to be slightly modified in order to apply this well known

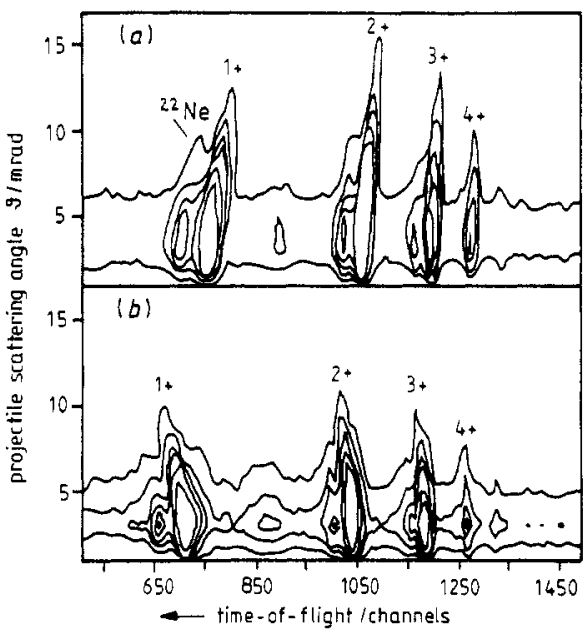

Figure 4. Tof plotted against the projectile scattering angle $\vartheta$ : the different kinematic branches of figure 3 are separated by setting conditions on the projectile azimuthal scattering angle (see also figure 1 and text). 
technique, which is commonly used for the position readout of microchannel plate detector systems (Martin et al 1981). Two features have to be achieved: first, the avalanche must be sufficiently extended and cover several 'wedge-and-strip' segments (each $2 \mathrm{~mm}$ broad) of the anode to guarantee a reasonable determination of the centre of the charge distribution in the direction of discrete geometry ('strips'). Second, the signal to noise ratio has to be of the order of $300: 1$ to obtain a $0.2 \mathrm{~mm}$ resolution over a total active area of $50 \times 50 \mathrm{~mm}^{2}$ and a reasonable energy resolution has to be achieved. Therefore, the active volume of the counter, filled with isobutane at a pressure between $1 \times 10^{3}$ and $2 \times 10^{3} \mathrm{~Pa}$, was designed as a stack of three regions, separated by grids with a transmission of $71 \%$. As shown in figure 5 , the gaps between the first two grids were $2 \mathrm{~mm}$ each, the wedge and strip anode was set $8 \mathrm{~mm}$ behind the last grid. Since only few primary electrons are produced by fast protons, the first gap was used to collect those electrons and therefore only a small electric field of $\Delta V_{1}=100 \mathrm{~V}$ has been applied, not strong enough to initialise an avalanche process. Those electrons are then amplified by a factor of about $10^{6}$ to $10^{7}$ within the second gap, the avalanche region of the detector $\left(\Delta V_{2}=400 \mathrm{~V}\right)$. Depending on the actual conditions, the region between the third grid and the anode was either used as a drift path only with a small voltage applied in order to broaden the avalanche or was supplied with a higher voltage to achieve further amplification. The combination of a 'collection' and 'amplification' part of the detector provided high pulses with a good signal to noise ratio as well as considerable energy resolution of about $30 \%$ for $1 \mathrm{MeV}$ protons. By modifying the voltage between the third grid and the anode, the extension of the charge cloud could be controlled. Under optimised conditions, a position resolution of less than $0.2 \mathrm{~mm}$ over $50 \mathrm{~mm}$ in both directions and an average resolution of about $0.4 \mathrm{~mm}$ through long running times could be obtained at typical rates of 5000 up to 10000 particles per second.

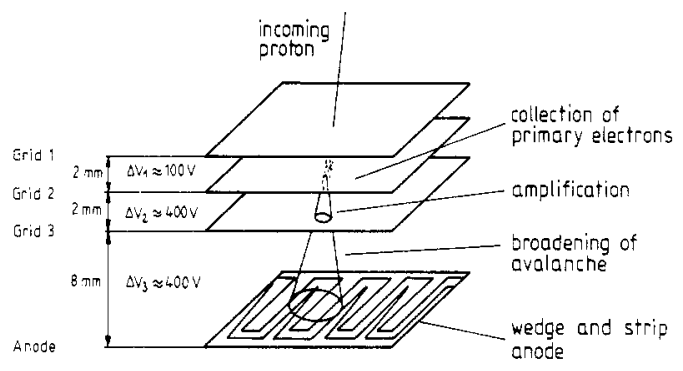

Figure 5. Schematic of the parallel-plate avalanche detector used for the two-dimensional position determination of the scattered projectiles.

\section{Data analysis}

\subsection{Calculation of the impact parameter}

Since scattering angles $\vartheta$ corresponding to impact parameters up to roughly $0.2 a_{0}$ have been investigated, a screened Coulomb potential with a screening parameter of

$$
a=0.8 a_{0}\left[\left(Z_{\mathrm{P}}^{1 / 3}+Z_{\mathrm{T}}^{1 / 3}\right)\right]^{1 / 2}
$$


( $a_{0}$ is the Bohr parameter, $Z_{\mathrm{P}}, Z_{\mathrm{T}}$ are the projectile and target nuclear charges) has been used to calculate $b$. The values obtained differ by up to $10 \%$ from the results of a calculation with an unscreened Rutherford potential at the largest impact parameters. They are in good agreement with СТMC results which need no additional assumptions about the scattering potential to obtain the $b(\vartheta)$ relationship since all mutual interactions between all particles except the electron-electron interaction are included.

\subsection{Relative multiple ionisation probabilities}

Having set appropriate windows on the azimuthal projectile scattering angle $\varphi_{\mathrm{P}}$ to ensure equal detection solid angles for recoil-ions of different energies and charge states, relative multiple ionisation probabilities $P_{q}^{\prime}(b)$ at a distinct $b$ can easily be obtained from the number of true coincidences for a certain charge state $N_{q}(b)$ divided by the number of randomly scattered particles $N_{\text {ran }}(b)$ at the same $b$ :

$$
P_{q}^{\prime}(b)=N_{q}(b) / N_{\text {ran }}(b) \text {. }
$$

\subsection{Absolute normalisation}

Since the relative probabilities for the charge state $q=1$ were found to be constant in very good approximation within the investigated $b$ regime, the absolute normalisation could be obtained using absolute single ionisation cross sections $\sigma_{1}$ by DuBois and Manson (1987) applying the following procedure.

The total number of recoil ions $N_{1}(\Delta b)$ of charge state $q=1$ in the $b$ range $b_{1} \leqslant b \leqslant b_{2}$ is given by:

$$
N_{1}(\Delta b)=N_{0}\left(\Delta \Omega_{1} / 4 \pi\right) \varepsilon \Delta x 2 \pi \int_{b_{1}}^{b_{2}} P_{1}(b) b \mathrm{~d} b
$$

where $N_{0}$ is the number of incoming projectiles, $\Delta x$ the target thickness, $\Delta \Omega_{1} / 4 \pi$ is the effective solid angle and $\varepsilon$ the detection efficiency of the system. The total number of produced recoil ions $N_{1}$ can be written as:

$$
N_{1}=N_{0}\left(\Delta \Omega_{2} / 4 \pi\right) \varepsilon \Delta \times 2 \pi \sigma_{1} .
$$

For the ratio one obtains:

$$
N_{1}(\Delta b) / N_{1}=\left(\left(\Delta \Omega_{1} / \Delta \Omega_{2}\right) 2 \pi \int_{b_{1}}^{b_{2}} P_{1}(b) b \mathrm{~d} b\right) / \sigma_{1} .
$$

Due to the constant probability within $\Delta b$ the integration leads after some rearrangement to the equation:

$$
P_{1}(b)=\left(N_{1}(\Delta b) / N_{1}\right) \sigma_{1}\left(2 \pi\left(b_{2}^{2}-b_{1}^{2}\right)\right)^{-1}\left(\Delta \Omega_{2} / \Delta \Omega_{1}\right) .
$$

All numbers on the right-hand side of equation (6) are experimentally determined, $\left(\Delta \Omega_{1} / \Delta \Omega_{2}\right)$ being the ratio of the actual $\varphi_{\mathrm{P}}$ window to $2 \pi$. Since the ratios $P_{q>1}(b) / P_{1}(b)$ obtained by the evaluation of relative multiple ionisation cross sections are known, all $P_{q}(b)$ are absolutely normalised by this procedure.

The total error in the $P_{q}(b)$ is estimated to be about $35 \%$. The contributions to the total error are: uncertainties in the absolute cross section $\sigma_{1}$ of $30 \%$, in the determination of $\left(b_{2}^{2}-b_{1}^{2}\right)$ of $10 \%$, in the determination of the total number of recoil ions of $8 \%$ and of that in the interval $\Delta b$ of $9 \%$. The given $b$ values should be accurate within $5 \%$. Only statistical errors are indicated in the figures, which is the total error for the relative $P_{q}(b)$. 


\section{Results and discussion}

\subsection{Multiple ionisation probabilities}

In figure $6(a, b)$ the measured absolute multiple ionisation probabilities are shown for $0.5 \mathrm{MeV}$ and $1 \mathrm{MeV}$ p on $\mathrm{Ne}$ collisions. Since for both energies the projectile velocity $v_{\mathrm{P}}$ is larger than the mean velocity of the $\mathrm{Ne}$ L-shell electrons $v_{\mathrm{e}}$ by a factor of more than three and, in addition, those electrons should give the main contribution for the production of the lower charge states, a decrease of the total ionisation probabilities with increasing projectile velocity is expected. This behaviour is clearly reflected in the experimental data: at $0.5 \mathrm{MeV}$ the total ionisation probability (sum over all charge states) is $41 \%$ and decreases to a value of $26 \%$ at $1 \mathrm{MeV}$ proton impact. Within the experimental error bars these values are in good agreement with theoretical results of CTMC calculations of $40 \%$ at $0.5 \mathrm{MeV}$ and $28 \%$ at $1 \mathrm{MeV}$. The calculations have been performed at two different impact parameters and show an impact parameter dependence in accordance with the experimental data.
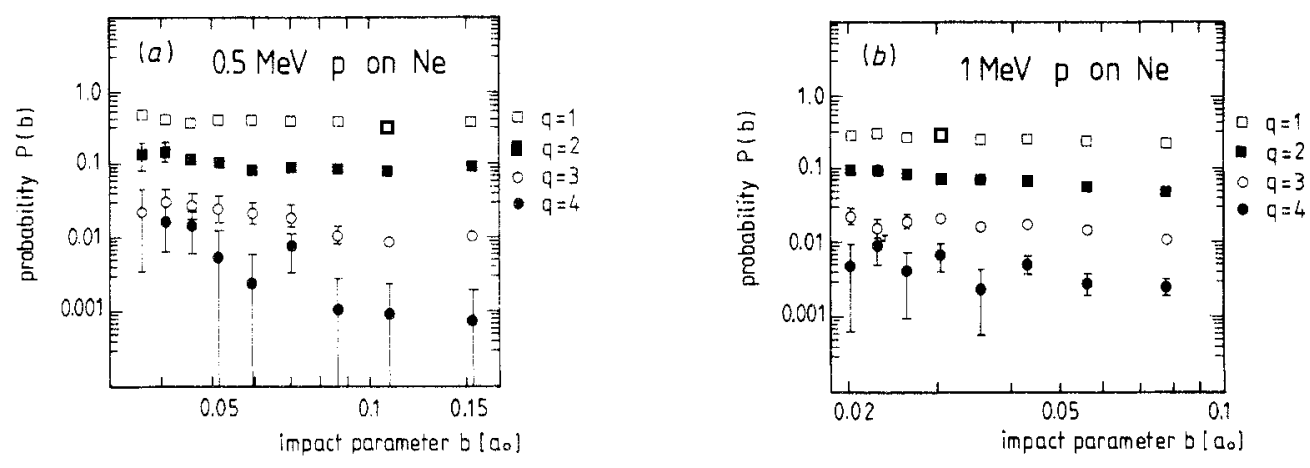

Figure 6. Multiple ionisation probabilities for $0.5 \mathrm{MeV}(a)$ and $1 \mathrm{MeV}(b) \mathrm{p}$ on $\mathrm{Ne}$ in dependence on the impact parameter. The recoil-ion charge states are indicated in the figure.

Ne charge states up to $q=4$, produced in a single encounter with the proton, could experimentally be identified. In contrast to the nearly $b$-independent shape of the single and double ionisation probabilities, which imply constant $\mathrm{L}$ and $\mathrm{K}$-shell ionisation probabilities $P_{\mathrm{L}}(b)$ and $P_{\mathrm{K}}(b)$, the $P_{q}(b)$ for $q=3,4$ increase with decreasing impact parameter implying an increase of $P_{\mathrm{L}}(b)$ or $P_{\mathrm{K}}(b)$. This behaviour, which is more pronounced at $0.5 \mathrm{MeV}$ proton impact, indicates deviations from an independentelectron model and the resulting binomial distribution of the $P_{q}(b)$.

\section{2. $K$ and $L$-shell ionisation probabilities}

To investigate this behaviour more quantitatively, $\mathrm{L}$ - and $\mathrm{K}$-shell ionisation probabilities have been derived from the experimental ratios $P_{q}(b) / P_{q=1}(b)$ at two impact parameters for both collision energies. This analysis can only be considered as an attempt to extract the overall features of multiple ionisation, since many different processes apart from direct ionisation contribute to multiple ionisation probabilities: e.g. electron transfer to the projectile (electron capture), autoionisation of multiply excited states as well as core relaxation mechanisms ('shake off'). All these contributions have not explicitly been included. 
Neglecting electron capture seems to be justified because the total cross section is about three orders of magnitude smaller than the related ionisation cross section (Tawara and Russek 1973, Eckhard and Schartner 1983). The relative contribution of the electron capture channel to total ionisation probabilities, however, might be strongly enhanced at the small impact parameters investigated. Therefore, in spite of negligibly small total capture cross sections, it cannot be excluded that significant contributions of electron capture might occur. Since there are no $b$-dependent experimental data available and $n$ CTMC calculations, which in principle could separate between the different processes, are extremely time consuming, no quantitative estimate of this effect can be given here.

Also little experimental as well as theoretical information exists on the contribution of core relaxation processes (shake off) to multiple ionisation of Ne for proton impact. Total cross section studies indicate (Haugen et al 1982) that the fraction of $\mathrm{Ne}^{2+}$ due to shake off is below $3 \%$. Since we observe ratios of $\mathrm{Ne}^{2+} / \mathrm{Ne}^{+}$between $25 \%$ and $50 \%$ shake off should be of minor importance for the $\mathrm{Ne}^{2+}$ production. However for close collisions, similar to the case of electron capture, shake off following $\mathrm{K}$-shell ionisation might considerably enhance the production of $\mathrm{Ne}^{3+}$ and $\mathrm{Ne}^{4+}$ recoil ions. Even for this process no quantitative information for charged particle impact is available at present.

Estimates on the basis of $n$ CTMC calculations (Berg et al 1988) indicate that autoionisation after multiple excitation of the $\mathrm{Ne}$ L-shell yield a contribution to the total ionisation probability between $10 \%$ and $20 \%$.

Having in mind the neglect of all those contributions, the probability to produce for instance a singly charged $\mathrm{Ne}$ target ion $P_{1}(b)$ can be written as (McGuire and Weaver 1977):

$$
\begin{aligned}
P_{1}(b)=\left(\begin{array}{l}
8 \\
1
\end{array}\right) & P_{\mathrm{L}}(b)^{1}\left(1-P_{\mathrm{L}}(b)\right)^{7}\left(1-P_{\mathrm{K}}(b)\right)^{2} \\
& +\left(\begin{array}{l}
8 \\
0
\end{array}\right) P_{\mathrm{L}}(b)^{0}\left(1-P_{\mathrm{L}}(b)\right)^{8} 2 P_{\mathrm{K}}(b)\left(1-P_{\mathrm{K}}(b)\right)(1-\omega)
\end{aligned}
$$

where $\left(\begin{array}{l}8 \\ 1\end{array}\right)$ and $\left(\begin{array}{l}8 \\ 0\end{array}\right)$ are the binomial coefficients. It is assumed that a $\mathrm{K}$ vacancy will stabilise via Auger decay with the fluorescence coefficient $\omega$ resulting in an emission of one further electron. As is usually done in similar calculations, all L-shell electrons were treated equally, which is a further simplification since L-subshell ionisation cross sections differ significantly due to the different binding energies of the respective electrons.

A singly charged $\mathrm{Ne}$ atom can be obtained from single L-shell ionisation times the probability that no K-shell ionisation took place $\left(1-P_{\mathrm{K}}(b)\right)^{2}$, which is the first part of equation (7) plus the probability that no L-shell ionisation occurred but the K-shell was singly ionised and stabilises via radiative decay with a probability of $(1-\omega)$. From the ratio $P_{q}(b) / P_{q=1}(b)$ for each combination of charge states $q=1$ and $q$ one can derive a one-dimensional set of solutions $P_{\mathrm{K}}$ and $P_{\mathrm{L}}$ in the two dimensional $P_{\mathrm{K}}-P_{\mathrm{L}}$ plane using binomial statistics. In our case, due to the statistical errors in the experimental multiple ionisation probabilities, one obtains a two dimensional subset of solutions in the $P_{\mathrm{K}}-P_{\mathrm{L}}$ plane. For $0.5 \mathrm{MeV}$ p on Ne these subsets of $P_{\mathrm{K}}-P_{\mathrm{L}}$ combinations are shown in figure $7(a, b)$ for the experimental ratios $P_{2} / P_{1}, P_{3} / P_{1}$ and $P_{4} / P_{1}$ at two different impact parameters of $0.053 a_{0}$ and $0.078 a_{0}$. A further condition can be derived from the measured total ionisation probability with $P_{\text {tot }}=8 P_{\mathrm{L}}+2 P_{\mathrm{K}}(1-\omega)$ allowing 
only for $P_{\mathrm{K}}-P_{\mathrm{L}}$ combinations on the left-hand side of the straight line. Since only ratios of probabilities are needed, all systematical errors in the absolute normalisation of the data cancel.

Under the assumption of completely independent electrons, these subsets have one common section which determines the unique solution $P_{\mathrm{K}}, P_{\mathrm{L}}$ within statistical error bars. As is obvious from figure $7(a, b)$, only for the smaller impact parameter investigated such a $P_{K}-P_{L}$ combination could be found mainly due to the larger error bars at small $b$. At the large $b$ value, however, a strong systematical deviation from the statistical behaviour in particular for the ratio $P_{4} / P_{1}$ is observed for both proton energies: higher charge states $q=3,4$ are produced with a higher probability than expected from the IPA.

Deviations from binomial statistics might be expected due to the fact that the ionisation potential increases with the degree of ionisation in such a way that the calculation of the single ionisation probability for a given electron shell is questionable. This effect should lead to an overestimation of the probabilities for higher charge states and therefore cannot be the reason for the observed discrepancies. As has been discussed above, contributions to the $P_{3,4}(b)$ in connection with electron capture might become important at small impact parameters. Due to the strong decrease of the total capture cross sections with the projectile velocity this influence should be of major importance at low velocities whereas we observe the largest deviations at high energies. On the contrary the relative contribution of shake-off processes following K-shell ionisation should increase with increasing velocity in the velocity regime investigated (McGuire 1982) so that the observed deviations might be due to core relaxation processes.
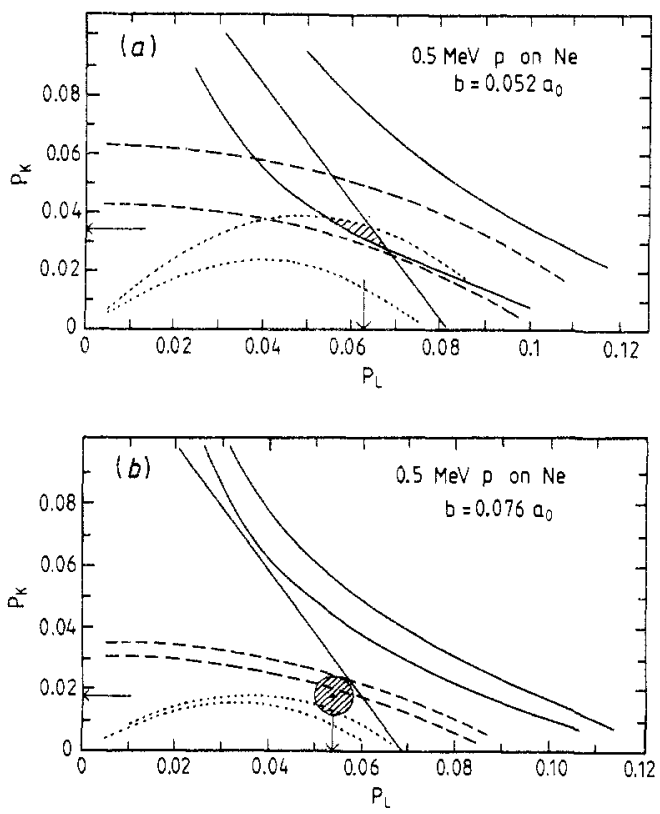

Figure 7. L-shell $\left(P_{\mathrm{L}}\right)$ plotted against K-shell $\left(P_{\mathrm{K}}\right)$ ionisation probability for $0.5 \mathrm{MeV} \mathrm{p}$ on $\mathrm{Ne}$ at two different impact parameters. The areas between the different line types give possible $P_{L}-P_{K}$ combinations derived from the experimental ratios $P_{q^{\prime}} / P_{q}$ for $q^{\prime}=4, q=1$ (full curves), $q^{\prime}=3, q=1$ (broken curves) and $q^{\prime}=2, q=1$ (dotted curves). Shaded area: overlap region. 
Despite deviations from an exact statistical behaviour K- and L-shell ionisation probabilities have been extracted from the experimental data for both projectile energies at two different impact parameters. In table 1 these data are compared with CTMC, SCA and experimental values (Horsdal-Pedersen et al 1982). CTMC results are only given for $P_{L}$ and for the larger impact parameter at both energies: due to the high velocity of $\mathrm{Ne} \mathrm{K}$-shell electrons compared with the projectile velocities at the investigated energies theory is at the limits of its regime of validity for the calculation of $P_{\mathrm{K}}$. Calculations at small impact parameters are extremely time consuming and could not be performed within available computing times. The theoretical predictions for L-shell ionisation probabilities are in surprisingly good accordance with the experimental values. Our extracted $\mathrm{K}$-shell ionisation probabilities agree within about $50 \%$ with the directly measured $P_{\mathrm{K}}$ of Horsdal-Pedersen et al (1982) and calculations in the semiclassical approximation (sCA, Trautmann et al 1982). SCA results for $P_{\mathrm{L}}(b)$ strongly underestimate the experimental results indicating that the perturbation of the Ne L-shell by the incoming proton is too strong in order to apply perturbation theory. In summary, theoretical and experimental results by other authors are in reasonable agreement with the experimental values at both energies and both impact parameters indicating that, apart from obvious deviations from an exact statistical behaviour, again the IPA shows itself to be a reasonable approximation to describe the overall features of the complicated multiple ionisation process.

Table 1. Experimentally derived L- and $\mathrm{K}$-shell single ionisation probabilities in comparison with CTMC (Olson 1988) and SCA (Trautmann et al 1982) calculations. HP denotes experimental data of Horsdal-Pedersen et al (1982) who measured $P_{\mathrm{K}}(b)$ directly via differential Auger spectroscopy.

\begin{tabular}{|c|c|c|c|c|}
\hline \multirow{3}{*}{$\begin{array}{l}\text { Exp. } \\
\text { CTMC }\end{array}$} & \multicolumn{2}{|c|}{$b=0.08 a_{0}$} & \multicolumn{2}{|c|}{$b=0.05 a_{0}$} \\
\hline & $1.8 \%$ & $5.3 \%$ & $3.3 \%$ & $6.3 \%$ \\
\hline & - & $6.0 \%$ & - & - \\
\hline SCA & $1.9 \%$ & $1.6 \%$ & $2.4 \%$ & $1.7 \%$ \\
\hline HP & $1.7 \%$ & - & $2.1 \%$ & - \\
\hline \multicolumn{5}{|c|}{$E_{\mathrm{P}}=1000 \mathrm{keV}$} \\
\hline & \multicolumn{2}{|c|}{$b=0.08 a_{0}$} & \multicolumn{2}{|c|}{$b=0.04 a_{0}$} \\
\hline Exp. & $1.7 \%$ & $3.2 \%$ & $2.5 \%$ & $4.1 \%$ \\
\hline CTMC & - & $3.7 \%$ & - & - \\
\hline $\mathrm{SCA}$ & $2.0 \%$ & $0.8 \%$ & $2.8 \%$ & $0.9 \%$ \\
\hline $\mathrm{HP}$ & $2.7 \%$ & - & $3.3 \%$ & - \\
\hline
\end{tabular}

\section{Conclusion}

We have measured absolute multiple ionisation probabilities in dependence on the impact parameter for 0.5 and $1 \mathrm{MeV}$ p on $\mathrm{Ne}$ collisions. A PPAD was used in combination with a 'wedge-and-strip' anode readout to determine simultaneously the polar and azimuthal scattering angle of the projectile. 
The experimental data were analysed in the framework of the IPA and deviations from an exact statistical behaviour have been observed: highly charged $\mathrm{Ne}$ recoil ions $(q=3,4)$ are produced with higher probabilities than those emerging from pure statistical behaviour. However, the experimentally extracted $P_{\mathrm{L}}(b)$ are in good agreement with CTMC results showing that the overall behaviour of multiple ionisation is well described by the IPA. K-shell ionisation probabilities are reasonably well reproduced by SCA calculations.

\section{Acknowledgment}

Financial support by the 'Gesellschaft für technische Zusammenarbeit', GTZ, Eschborn, is gratefully acknowledged.

\section{References}

Åberg T and Goscinski A 1982 X-Ray and Atomic Inner-Shell Physics ed B Crasemann (New York: AIP) p 121

Becker R L, Ford A L and Reading J F 1984 Phys. Rev. A 293111

Berg H et al 1988 J. Phys. B: At. Mol. Opt. Phys. 213929

Cocke C L 1979 Phys. Rev. A 20749

Dörner R, Ullrich J, Schmidt-Böcking H and Olson R E 1989 Phys. Rev. Lett. 63147

DuBois R D and Manson S T 1987 Phys. Rev. A 352007

Eckhard M and Schartner K H 1983 Z. Phys. A 312321

Gaukler G, Schmidt-Böcking H, Schuch R, Schulè R, Specht H J and Tseruya I 1977 Nucl, Instrum. Methods 141115

Giese J P and Horsdal E 1988 Phys. Rev. Lett. 602018

Grandrin G P, Hennecart D, Husson X, Lecler D, Lesteven-Vaisse I and Lisfi D 1988 Europhys. Lett. 6683

Haugen K H, Andersen L H, Hvelplund P and Knudsen H 1982 Phys. Rev. A 261962

Horbatsch M 1986 Z. Phys. D 1337

1989 J. Phys. B: At. Mol. Opt. Phys. 22 L639

Horbatsch M and Dreizler R 1985 Phys. Lett. 113A 251

Horsdal-Pedersen E, Folkmann F and Pedersen N H 1982 J. Phys. B: At. Mol. Phys. 15739

Kamber E Y, Cocke C L, Cheng S and Varghese S L 1988 Phys. Rev. Lett. 602026

Kelbch S, Schmidt-Böcking H, Ullrich J, Schuch R, Justiniano E, Ingwersen H and Cocke C L 1984 Z. Phys. A 3179

Kelbch S, Cocke C L, Hagmann S, Horbatsch M, Kelbch C, Koch R and Schmidt-Böcking H 1988 Phys. Lett. 127A 92

Levin J C, Short R T, Cederquist C O H, Elston S B, Gibbons P, Sellin I A and Schmidt-Böcking H 1987 Phys. Rev. A 371649

Martin C, Jenlinsky P, Lampton M, Malina R F and Anger H O 1981 Rev. Sci. Instrum. 521067

McGuire J H 1982 J. Phys. B: At. Mol. Phys. 17 L779

McGuire J H and Weaver L 1977 Phys. Rev. A 1641

Müller A, Schuch B, Groh W, Salzborn E, Beyer H F, Mokler P H and Olson R E 1986 Phys. Rev. A 333010

Olson R E 1979 J. Phys. B; At. Mol. Phys. 121843

Olson R E 1988 Electronic and Atomic Collisions ed H B Gilbody, W R Newell, F H Read and A C H Smith (Amsterdam: Elsevier) pp 271-85

Olson R E, Ullrich J and Schmidt-Böcking H 1987 J. Phys. B: At. Mol. Phys. 20 L809

1989 Phys. Rev. A 395572

Schuch R, Schöne H, Miller P D, Krause H F, Dittner P F, Datz S and Olson R E 1988 Phys. Rev. Lett. 60 925

Tawara H and Russek A 1973 Rev. Mod. Phys. 45178

Trautmann D, Rösel F and Baur G 1982 Nucl. Instrum. Methods 169121 
Ullrich J, Bethge K, Kelbch S, Schadt W, Schmidt-Böcking H and Stiebing K E 1986 J. Phys. B: At. Mol. Phys. 19437

Ullrich J, Horbatsch M, Dangendorf V, Kelbch S and Schmidt-Böcking H 1988 J. Phys. B: At. Mol. Opt. Phys. 21611

Ullrich J, Olson R E, Dörner R, Dangendorf V, Kelbch S, Berg H and Schmidt-Böcking H 1989 J. Phys. B: At. Mol. Opt. Phys. 22627 